\title{
The benefit of an acute stroke unit in patients with intracranial haemorrhage: a controlled trial
}

\author{
O M Rønning, B Guldvog, K Stavem
}

\begin{abstract}
Objectives-Patients with stroke receiving organised inpatient (stroke unit) care after stroke are more likely to be alive and independent compared with patients offered conventional care. The objective was to determine the effect of an acute stroke unit on patients with primary intracranial haemorrhage.

Methods-In a prospective controlled study, the effect of an acute stroke unit was examined on 30 day and 1 year mortality in patients with primary intracranial haemorrhage. Patients treated in general medical wards served as controls.

Results-Of 121 patients included, 56 were allocated to an acute stroke unit and 65 to a general medical ward. The 30 day mortality rate was $39 \%$ in the acute stroke unit compared with $63 \%$ in the general medical wards, and the 1 year mortality rates were $52 \%$ and $69 \%$, respectively. There was a difference between the 30 day and 1 year survival curves between the groups $(p=0.007$ and 0.013 , respectively); however, there was no difference in survival between 30 and 365 days. There was no difference in risks of being discharged home or to long term care between the groups.

Conclusions-In this study admission to an acute stroke unit reduced mortality $\mathbf{3 0}$ days and 1 year after primary intracranial haemorrhage, which could be attributed to a large difference in survival during the first 30 days.

(F Neurol Neurosurg Psychiatry 2001;70:631-634)
\end{abstract}

Keywords: stroke unit; intracranial haemorrhage; mortality

Department of

Neurology, Central

Hospital of Akershus,

1474 Nordbyhagen,

Norway

O M Rønning

Foundation for Health Services Research

O M Rønning

B Guldvog

K Stavem

Department of

Medicine

K Stavem

Correspondence to:

Dr O M Rønning

omro@online.no

Received 24 January 2000

and in final form

4 December 2000

Accepted 19 December 2000 of the type of stroke. We have previously reported the results of two controlled studies on the effect of an acute stroke unit on survival and dependency. ${ }^{11}{ }^{12}$ A subgroup analysis of 115 of these patients with intracerebral or cerebellar haemorrhage treated in an acute stroke unit or general medical ward indicated a benefit of an acute stroke unit for patients with primary intracranial haemorrhage. ${ }^{11}$ In this study, we wanted to confirm the effect of an acute stroke unit on 30 day and 1 year mortality in a prospective study with a different sample of patients with primary intracranial haemorrhage. We hypothesised that the prognosis of patients with primary intracranial haemorrhage is related to whether they are treated in an acute stroke unit or in general medical wards.

\section{Subjects and methods}

This study was a prospective parallel cohort study with two groups. The design of the study is described in detail elsewhere. ${ }^{112} \mathrm{~A}$ consecutive series of 135 patients 60 to 85 years of age were admitted to hospital with acute primary intracranial haemorrhage in the study period from February 1995 to December 1997. Patients were eligible if they had an intracerebral or cerebellar haemorrhage verified by CT. If patients had haemorrhage, a CT performed more than 24 hours after the start of symptoms did not exclude them from this study provided that the other inclusion criteria were fulfilled. Exclusion criteria included symptoms of stroke $>24$ hours before admission to hospital, subarachnoid haemorrhage, subdural haemorrhage, and age $<60$ years as all patients under the age of 60 were treated in the stroke unit. The CT was interpreted by radiologists who were not involved in this study. None of the patients were included more than once. After inclusion, patients were allocated to treatment either in an acute stroke unit or in general medical wards. The selection of patients to either treatment arm was based on the two first digits in the birth number (day of the month). Patients born on the $1 \mathrm{st}-15$ th day of the month were allocated to the stroke unit. The decision to allocate patients to the different departments based on date of birth was determined by the hospital management independently of this study, because of a shortage of beds in the acute stroke unit. In practice, when the 10 beds were occupied, patients with stroke were still allocated to stroke unit treatment, because the 10 bed stroke unit in the department of neurology could expand on demand at the expense of the general neurological beds. The Central Hospital was the only hospital in the catchment area that received patients with 
stroke, and general practitioners in the area were asked to refer all patients with this diagnosis immediately to the hospital. If a stroke was suspected relatives could call directly to an emergency number for an ambulance to the hospital. The primary study outcomes were deaths from any cause. Secondary outcomes were number of days in hospital and proportion of the patients alive and discharged to their homes. Deaths were recorded from the $\mathrm{Na}$ tional Norwegian Population Register, an official nationwide register containing name, date of birth, address, and date of death. The patient or their next of kin gave informed consent to be treated in hospital. The trial was approved by a regional ethics committee and a reference group at the hospital.

\section{TREATMENT IN THE ACUTE STROKE UNIT}

An acute stroke unit is generally defined as a stroke unit accepting patients acutely but with early discharge (usually $<1$ week). The conservative care of patients with primary intracranial haemorrhage in the acute stroke unit was mainly the same as for patients with cerebral infarctions. Fluid management was provided with isotonic solutions not containing glucose given intravenously $(50-100 \mathrm{ml} / \mathrm{h}$ for the first 24 hours for most patients). If systolic blood pressure was greater than 230 or diastolic blood pressure was greater than 120 on two readings the patient was considered for antihypertensive treatment. In our stroke unit acute hypertension in a patient with primary intracranial haemorrhage was initially treated with repeated intramuscular injections of $12.5 \mathrm{mg}$ chlorpromazine. We could often control high blood pressure among anxious or agitated patients with this medication. If rectal temperature was greater than $38.0^{\circ} \mathrm{C}$, the patient was treated with paracetamol $(1-3 \mathrm{~g} / 24 \mathrm{~h})$ if necessary. When a repiratory tract infection was detected or suspected intravenously penicillin treatment was started. Extensive physiotherapy was initiated to prevent deep vein thrombosis and low molecular weight heparin was seldom prescribed even among patients with paralysis. Patients without reduced consciousness or extensive intraventricular rupture of blood were mobilised out of bed within the first 24 hours and activation and rehabilitation was started as soon as possible. Intracranial pressure was not monitored. Blood gas analysis was performed to correct hypoxia and hypercapnia. All patients were considered for possible neurosurgical intervention using the following criteria for referral to surgery: cerebellar haemorrhage greater than $3 \mathrm{~cm}$ and/or signs of deterioration or supratentorial haemorrhage between 3 and $6 \mathrm{~cm}$ and signs of deterioration. Our stroke unit was organised according to the principles of organised inpatient care of patients with stroke. ${ }^{10}$

TREATMENT IN THE GENERAL MEDICAL WARDS Treatment in the general wards in the department of medicine was typically neither based on specific guidelines nor organised in a multidisiplinary team. Patients were treated according to good medical practice at the time. They were immobilised for up to 1 week before rehabilitation was started. Primary prevention of deep vein thrombosis was routinely provided with low molecular weight heparin. There were no written guidelines for treatment of stroke fever, moderately raised blood glucose, registration of complications, or response to these complications. In the medical wards procedure for treatment of patients with stroke the staff was instructed to be aware of the following complications: urinary retention, bed sores, aspiration, and infections.

\section{STATISTICAL ANALYSIS}

For descriptive statistics we used the MannWhitney $U$ test or $\chi^{2}$ test. We estimated survival by the Kaplan-Meier method. Comparison of survival curves was performed with Breslow's generalised Wilcoxon test. Multivariate Cox regression analysis was used to adjust relative risks in the survival analysis for imbalance in conscious level, prior stroke, and diabetes. We compared the risk of death, in hospital death, and discharge to home, long term care, or long term rehabilitation between the two groups, presenting odd ratios (ORs) with 95\% confidence intervals (95\% CIs). Because of imbalance in the baseline characteristics, we used multivariate logistic regression analysis to obtain adjusted ORs with $95 \%$ CIs. A study with 60 patients in each group has a $90 \%$ power to detect an absolute risk reduction of $30 \%$ in number of deaths at 1 year, with a $p$ value $<0.05$.

\section{Results}

Between 1 February, 1995 and 31 December 1997, 135 patients were eligible for the two treatment groups: 62 to the acute stroke unit and 73 to a general medical ward. Of these, 14 patients (six in the acute stroke unit and eight in the general medical ward) were admitted to hospital $>24$ hours after stroke and hence did not meet the eligibility criteria. Two patients with date of birth between the 1st and 15th of a month were treated in the general medical ward, but they were analysed in the acute stroke unit group. Demographic data and prior

Table 1 Characteristics of eligible patients with stroke allocated to receive care in the stroke unit or general medical wards

\begin{tabular}{llll}
\hline Characteristics & Stroke unit & Medical wards & p Value \\
\hline Mean age (y (SD)) & $76(7)$ & $76(7)$ & 0.60 \\
Female sex & $22 / 56(39)$ & $31 / 65(48)$ & 0.37 \\
Unconscious & $15 / 56(27)$ & $27 / 65(42)$ & 0.13 \\
Medical history: & & & \\
$\quad$ TIA & $8 / 56(14)$ & $9 / 65(14)$ & 1.00 \\
Prior stroke & $9 / 56(16)$ & $13 / 65(20)$ & 0.64 \\
Myocardial infarction & $7 / 56(13)$ & $8 / 65(12)$ & 1.00 \\
Angina pectoris & $13 / 56(23)$ & $8 / 65(12)$ & 0.15 \\
Atrial fibrillation & $5 / 56(9)$ & $6 / 65(9)$ & 1.00 \\
Hypertension & $18 / 56(32)$ & $17 / 65(26)$ & 0.54 \\
Diabetes & $3 / 56(5)$ & $6 / 65(9)$ & 0.50 \\
Malignancy & $4 / 56(7)$ & $4 / 65(6)$ & 1.00 \\
Current smoker & $10 / 56(18)$ & $13 / 65(20)$ & 0.82 \\
Time to hospital admission: & $14 / 54(26)$ & $18 / 62(29)$ & 0.44 \\
<3 h & $20 / 54(37)$ & $17 / 62(27)$ & 0.18 \\
3-6 h & $10 / 54(19)$ & $10 / 62(15)$ & 0.47 \\
6-12 h & $10 / 54(19)$ & $17 / 62(27)$ & 0.20 \\
12-24 h & & & \\
\hline
\end{tabular}

Data are number of patients with a given characteristic/total number of patients (\%), unless otherwise stated. $p$ Values are two tailed. TIA=Transient ischaemic attack 


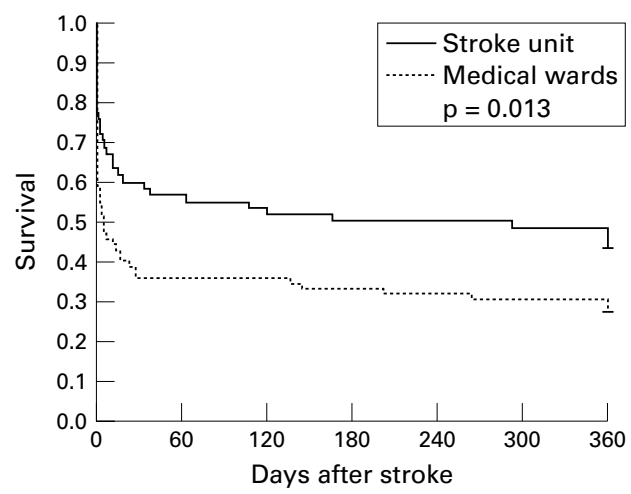

Figure 1 Survival after intracranial haemorrhage by treatment group $(n=121)$.

medical history did not differ between the two groups. However, there was a trend towards a higher number of patients with more severe strokes, prior stroke, diabetes, and later admission to hospital in the control group (table 1). Complete follow up was obtained until death or until December 1998. After an initial higher case fatality rate among patients in the general medical ward during the first weeks, the slope of the survival curves were nearly identical (figure 1). The difference between the survival curves for the two groups was significant in favour of the acute stroke unit during the first 30 days and during 1 year $(\mathrm{p}=0.007$ and 0.013 , respectively). There was no difference between the groups between 30 days and 1 year. When adjusting for baseline differences in conscious level at admission, prior stroke, and diabetes in a multivariate Cox regression analysis, there was still a difference in risk ratios for death at 30 days $(p=0.035)$ and 1 year $(p=0.048)$. Among patients allocated to the acute stroke unit, the 30 day mortality rate was lower than in the control group, even after adjustment for baseline imbalance, whereas there was no difference after 30 days (table 2). In addition, in the multivariate analysis there was no difference in the ORs for in hospital death, discharge home, long term care, or long term rehabilitation (table 2). Differences in time from stroke onset to CT and differences in treatment are shown in table 3 .

\section{Discussion}

This study provides evidence for the benefit of immediate admission to an acute stroke unit for patients with primary intracranial haemorrhage. The risk of early death was significantly reduced. No controlled study has previously been designed to evaluate the acute treatment of patients with intracranial haemorrhages in a stroke unit. Our results demonstrate that the benefits of a stroke unit are not restricted to patients with ischaemic strokes. These results extend the findings of other stroke unit studies that accepted patients with no alterations of consciousness to acute treatment and continued care for several weeks if necessary. ${ }^{10}$ These studies showed a reduction in deaths, increased independence in activities of daily living, and reduced need for long term care, but none of them included patients exclusively within 24 hours of stroke onset or included patients in coma. Previous hospital based studies showed that 30 day mortality was $30 \%-40 \%,{ }^{5-6}$ lower than the $50 \%$ reported in community based studies. ${ }^{7}$ The explanation for this difference is the probable exclusion of patients with the most severe strokes not referred to hospital or likely to die before arrival at hospital. In our study 30 day case fatality in the control group was similar to that in community based studies. It is likely that many of the most severe cases

Table 2 Outcome by treatment groups

\begin{tabular}{llllr}
\hline Time after initial stroke & Stroke unit & Medical wards & OR (95\% CI) & OR (95\% CI)* \\
\hline 1 month death & $22 / 56(39)$ & $41 / 65(63)$ & $0.38(0.18-0.79)$ & $0.40(0.17-0.94)$ \\
12 month death & $29 / 56(52)$ & $45 / 65(69)$ & $0.48(0.23-1.00)$ & $0.58(0.24-1.38)$ \\
Deaths between 1 month and 12 months & $7 / 34(20)$ & $4 / 24(17)$ & $1.30(0.33-5.04)$ & $1.14(0.26-4.94)$ \\
Death in hospital & $18 / 56(32)$ & $34 / 65(52)$ & $0.43(0.21-0.91)$ & $0.43(0.15-1.21)$ \\
Discharged home & $15 / 56(27)$ & $9 / 65(14)$ & $2.28(0.91-5.71)$ & $1.60(0.62-4.00)$ \\
Discharged to long term care & $19 / 56(34)$ & $16 / 65(25)$ & $1.57(0.71-3.47)$ & $1.72(0.69-4.29)$ \\
Discharged to long term rehabilitation & $4 / 56(7)$ & $6 / 65(9)$ & $0.76(0.20-2.83)$ & $0.62(0.10-3.70)$ \\
\hline
\end{tabular}

Data are number of patients with a given characteristic/total number of patients (\%). p Values are two tailed; OR=odds ratio; $95 \%$ $\mathrm{CI}=95 \%$ confidence interval. ${ }^{\star} \mathrm{OR}$ after adjustment for baseline imbalance (unconsciousness, prior stroke, diabetes).

Table 3 Time from stroke to CT examination and differences in treatment offered in the stroke unit and in general medical wards

\begin{tabular}{llll}
\hline Treatment & Stroke unit $(n=54)$ & Medical wards $(n=62)$ & OR (95\% CI) \\
\hline Median time in hospital (days (IQR)) & $7(3-13)$ & $5(2-11)$ & \\
Time between stroke and CT & & & \\
$\quad$ CT<6 h & $26 / 54(48)$ & $19 / 62(31)$ & $1.10(0.98-4.50)$ \\
CT between 6 and 12 h & $15 / 54(28)$ & $16 / 62(26)$ & $0.58(0.49-2.50)$ \\
CT between 12 and 24 h & $9 / 54(17)$ & $16 / 62(26)$ & $0.47(0.15-1.43)$ \\
CT $>$ 24 h & $5 / 54(9)$ & $11 / 62(17)$ & $1.02(0.37-2.87)$ \\
Referral to the neurosurgical department & $8 / 54(15)$ & $9 / 62(15)$ & $1.61(0.77-3.35)$ \\
Mobilisation within the first day after admittance & $29 / 54(54)$ & $26 / 62(42)$ & N/A \\
Parenteral fluid & $54 / 54(100)$ & $46 / 62(74)$ & $0.20(0.04-0.96)$ \\
Low molecular weight heparin & $2 / 54(4)$ & $10 / 62(16)$ & $2.86(0.96-8.55)$ \\
Oxygen treatment & $49 / 54(91)$ & $48 / 62(77)$ & $1.98(0.90-4.34)$ \\
Paracetamol & $22 / 54(41)$ & $16 / 62(26)$ & $1.17(0.38-3.58)$ \\
Penicillin & $7 / 54(13)$ & $7 / 62(11)$ & $2.67(0.93-7.69)$ \\
Antibiotics except penicillin & $12 / 54(22)$ & $6 / 62(10)$ & \\
\hline
\end{tabular}

Data are number of patients with a given characteristic/total number of patients (\%), unless otherwise stated. $p$ Values are two tailed. $\mathrm{IQR}=$ interquartile range; $\mathrm{OR}=$ odds ratio; $95 \% \mathrm{CI}=95 \%$ confidence interval; $\mathrm{N} / \mathrm{A}=$ not available. 
reached hospital and were included in this particular study because an evaluation and verification of the stroke diagnosis by a general practitioner was not necessary before transport to hospital. Our study did not include patients admitted more than 24 hours after onset of symptoms, and hence some less severe strokes were presumably not included. Most of the early deaths among patients with primary intracranial haemorrhage are due to the neurological consequences of the haemorrhage. ${ }^{7}$ The most important differences in treatment provided in the two departments that could theoretically influence early deaths were the early onset of mobilisation and rehabilitation, treatment of fever, and administration of antibiotics and fluids intravenously. We did not find differences in the proportion of deaths between 1 month and 12 months after stroke in the two groups. The secondary end points were dependent on the number of patients who survived and early case fatality. The relatively short stay in hospital in both groups is partly explained by the many deaths the first days after admittance. More patients died in the first 2 days in the general medical ward and hence influence the median duration of stay. Could the findings of this study be a result of the allocation procedure? The allocation procedure was settled by the hospital management because of shortage of beds in the stroke unit, even though, if the stroke unit was full, patients were admitted to the stroke unit because the unit could temporarily expand at the expense of the neurological ward. Hence, patients were not excluded or admitted to the general medical ward because of shortage of beds in the acute stroke unit. All patients admitted were included if they had an acute stroke, and they all had a primary intracranial haemorrhage verified by CT. The same radiologists interpreted the CT, and their interpretation should not be biased by the study as they did not know about the allocation procedure. None of the patients with a stroke could be withdrawn from the study because of knowledge of which department they would be treated on. Two patients with date of birth between 1 st and 15 th of a month were treated on the "wrong" ward but their outcomes were analysed in the acute stroke unit group corresponding to intention to treat analysis. In conclusion, an acute stroke unit reduces mortality after primary intracranial haemorrhage. The influence of the acute stroke unit on 30 day mortality was more pronounced than previously reported for patients with ischaemic strokes. ${ }^{11}$ However, the results should be interpreted with caution and some of the difference could be due to baseline imbalance between the groups. Particularly, the onset of mobilisation and aggressive prevention of complications differed between the treatment groups. We thus think that conservatively treated patients with intracranial haemorrhage should be offered the same organised treatment as patients with ischaemic strokes. Further research into what benefit patients with primary intracranial haemorrhage have from more aggressive early medical and surgical treatment of intracranial haemorrhages is still needed.

1 Kunitz SC, Gross CR, Heyman A. The pilot stroke data bank: definition, design and data. Stroke 1984;15:740.

2 Fogelholm R, Nuutila M, Vuorela AL. Primary intracerebral heamorrhage in the Jyuaskyla region, Central Finland, 1985-9: incidence, case fatality rate, and functional outcome. I Neurol Neurosurg Psychiatry 1992;55:546-52.

3 Sacco RL, Wolf PA, Bharucha NE, et al. The risk of subarachnoid and intracerebral haemorrhages in blacks as compared to whites. N Engl F Med 1992;326:733-6.

4 Subarachnoid and intracerebral haemorrhage: natural history, prognosis, and precursive factors in the Framingham study. Neurology 1984;34:847.

5 Broderick J. Intracerebral haemorrhage. In: Gorelick PB, Alter M, eds. Handbook of neuroepidemiology. New York, NY: Marcel Dekker, 1994:141-64.

6 Anderson C, Chakera T, Stewart-Wynne E, et al. Spectrum of primary intracranial haemorrhage in Perth, Western Australia, 1989-90: incidence and outcome. $\mathcal{F}$ Neurol NeuAustralia, 1989-90: incidence and
rosurg Psychiatry 1994;57:936-40.

7 Counsell C, Boonyakarnukul S, Dennis M, et al. Primary intracerebral heamorrhage in the Oxfordshire community intracerebral heamorrhage in the Oxfordshir
stroke project. Cerebrovasc Dis $1995 ; 5 ; 26-34$.

8 Broderick J, Brott T, Tomsick T, et al. Intracranial haemorrhage is more than twice as common as subarachnoid haemorrhage. F Neurosurg 1993;78:188-91.

9 Broderick JP, Adams HP, Barsan W, et al. Guidelines for the management of spontaneous intracerebral haemorrhage. A statement for healthcare professionals from a special writing group of the stroke council, American Heart Association. Stroke 1999;30:905-15.

10 Langhorne P, Dennis M, eds. Stroke units: an evidence based approach. London: BMJ Publishing Group, 1998.

11 Rønning OM, Guldvog B. Stroke units versus general medical wards, I: 12 and 18-month survival: a randomized controlled trial. Stroke. 1998;29:58-62.

12 Rønning OM, Guldvog B. Stroke units versus general medical wards, II: Neurological deficits and activities of daily living. Stroke. 1998;29:586-62. 